Extrapolation from a North American population (ACCESS) may not be valid. This retrospective pilot study aimed to evaluate the potential of a novel web-based registry, by recording demographic and clinical characteristics, follow-up and treatment.

Methods 199 potential patients were identified from specialist respiratory clinics at two hospitals. 135 case notes were reviewed. Data were extracted from first and follow-up visits with random data quality checks by at least one other author.

Results Of 135 patients, 55\% were female and 47\% Afro-Caribbean; mean age (years) was $43.7 \pm 12.3$ and patients had on average three follow-up visits per year. Presenting symptoms included cough (95\%), breathlessness (41\%), skin rashes $(22 \%)$, arthralgia $(15 \%)$, fatigue (19\%), eye symptoms (14\%) and fever (11\%). $14 \%$ had stage 0 chest radiographs, $46 \%$ stage I, $16 \%$ stage II, $15 \%$ stage III and $9 \%$ stage IV. $45 \%$ had raised serum angiotensin-converting enzyme at first visit. $80 \%$ had histological confirmation. Systemic corticosteroids were the commonest treatment (21.5\% after first visit). Cutaneous involvement was highest in females (64\%) and AfroCaribbeans (48\%). Nodular lesions were the most common; $77 \%$ had granulomas on skin biopsy. $94 \%$ of those with skin disease had other organ involvement and received on average three treatments (topical $77 \%$, prednisolone $64 \%$, and hydroxychloroquine $45 \%$ ). During follow-up, 73\% reported improvement. 20 patients had definite/ probable neurosarcoid (Zajicek criteria). Symptoms included parasthesiae $(45 \%)$, visual disturbance $(30 \%)$, headache $(30 \%)$, seizures $(10 \%)$ and anosmia $(5 \%)$. Four had a VIIth nerve palsy. Diagnosis was made on MRI in $95 \% ; 20 \%$ had CNS biopsies. $80 \%$ received prednisolone and $25 \%$ intravenous methylprednisolone. $15 \%$ were discharged after remission. Of the rest, $40 \%$ deteriorated during follow-up, $40 \%$ stabilised and $20 \%$ improved. Raised serum ACE was common in those with cutaneous and neurological disease.

Conclusions A novel web-based registry is a valuable method of recording clinical data in sarcoidosis. Additional use by other centres and the prospective recording of details of new cases could provide useful epidemiological data for the UK and drive future hypothesisbased studies.

\section{S55 PULMONARY FIBROSIS WITH AND WITHOUT CO-EXISTING CONNECTIVE TISSUE DISEASE: DOES IT AFFECT SURVIVAL?}

doi:10.1136/thx.2010.150938.6

${ }^{1} \mathrm{~V}$ Navaratnam, ${ }^{2} \mathrm{~N}$ Ali, ${ }^{1} \mathrm{CJP}$ Smith, ${ }^{1} \mathrm{~A}$ Fogarty, ${ }^{1} \mathrm{R}$ B Hubbard. ${ }^{1}$ Divisional of Epidemiology, University of Nottingham, Nottingham, UK; ${ }^{2}$ Queens Medical Centre, Nottingham University Hospitals Trust, Nottingham, UK

Introduction The impact of co-existing connective tissue disease on the survival of patients with pulmonary fibrosis remains unclear, since studies into the subject have so far yielded varying results. This study compares the survival of individuals with idiopathic pulmonary fibrosis (IPF) to those with connective tissue disease associated pulmonary fibrosis (CTD-PF) using data from The Health Improvement Network (THIN), a large general practice database in the UK.

Methods Incident cases of individuals with IPF and CTD-PF between the years 2000 and 2008 were identified. Survival analysis was performed on this dataset using Kaplan-Meier methods and Cox regression was used to compare mortality rates between patients with IPF and CTD-PF, adjusting for age and gender.

Results During the study period, there were 2208 incident cases of IPF, who were predominantly male $(64 \%)$ and had a mean age at time of diagnosis of 73.9 years (95\% CI 73.5 to 74.4 years). In comparison there were 318 incident cases of CTD-PF, of which the majority were female $(56 \%)$ and the mean age at time of diagnosis was 67.8 years (95\% CI 66.6 to 68.9). The commonest connective tissue disease associated with pulmonary fibrosis was rheumatoid arthritis (211 cases), followed by systemic sclerosis (39 cases). The

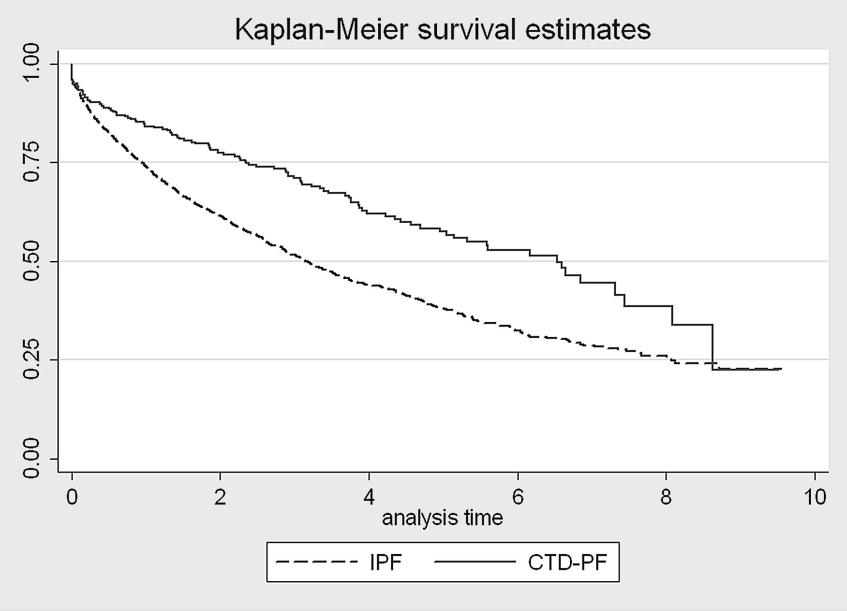

Abstract S55 Figure 1 Kaplan-Meier survival estimates.

mean follow-up period after diagnosis for our cohort was 2.4 person years and during this period 1117 (51\%) patients with IPF and 116 (36\%) patients with CTD-PF had died. The mortality rates for patients with IPF and CTD-PF were 221 (95\% CI 208 to 234) and 121 (95\% CI 101 to 146) deaths per 1000 person years respectively. The median survival for patients with CTD-PF was significantly higher at 6.5 years compared to 3.2 years in patients with IPF $(p=0.0033)$ (See Abstract S55 Figure 1). After adjusting for age and gender, patients with IPF had a worse prognosis compared to those with CTD-PF (HR 1.32, 95\% CI 1.08 to 1.60 ).

Conclusion Individuals who have CTD-PF have a better prognosis after diagnosis when compared to those with IPF. This may relate to the differing natural histories of the diseases and needs further investigation.

\section{S56 FLAT TRACHEA SYNDROME: AN UNDER-DIAGNOSED AND UNDER-TREATED CONDITION?}

doi:10.1136/thx.2010.150938.7

G Niranjan, J F K Marzouk. University Hospital of Coventry and Warwickshire, Coventry, UK

Objectives Flat Trachea Syndrome (Tracheobronchomalacia) is a central airway disease characterised by weakness of the wall and dynamic decrease in the tracheal lumen and the large bronchi, particularly while exhaling. It causes chronic symptoms such as cough, dyspnoea, increase in recurrent infections, and poor secretion management, but it can also progress to chronic respiratory failure and death. It remains largely under-diagnosed unless clinicians are thoroughly acquainted with its peculiar symptomology being usually confused with other common diseases like chronic obstructive pulmonary disease (COPD) or asthma. Surgery with posterior tracheobronchial splinting (tracheobronchoplasty) using a polytetrafluoroethylene (PTFE) Teflon patch has been considered as a possible treatment option for this condition.

Methods A retrospective series of 28 patients that underwent tracheobronchoplasty using a PTFE Teflon patch between July 1998 and July 2010 were evaluated for symptoms, lung capacity and exercise capacity.

Results The age range of patients treated was from 24 to 82 (mean 66). $61 \%$ (17) of those treated were men, with all cases being diagnosed with obstructive airways disease on spirometry, with the FEV1 ranging from $34 \%$ to $65 \%$ pre-operatively. 25 patients (89\%) presented with severe dyspnoea, 23 patients $(82 \%)$ with uncontrollable cough, and 21 patients $(75 \%)$ reported recurrent pulmonary infections. There was no in-hospital or 30-day mortality. After surgery symptomatic 
improvement in dyspnoea was reported in 24 of 28 patients $(p<0.001)$, with the cough disappearing in 19 of 21 patients $(p<0.001)$. At 3 months the FEV1 was $>80 \%$ in all 28 patients. The mean exercise capacity was improved in 17 (61\%) patients.

Conclusions The condition of tracheobronchomalacia is a misnomer and we propose the term "Flat Trachea syndrome" and/or "expiratory prolapse of the tracheobronchial posterior membrane". It is a rare but severely debilitating condition which can be diagnosed easily by an awake flexible bronchoscopy and dynamic biphasic inspiratory/expiratory CT. Surgical airway splinting with a PTFE (Teflon) patch considerably improves respiratory symptoms, quality of life and functional status in highly selected patients with this under-diagnosed and under-treated condition.

\section{S57 EVALUATION OF CIRCULATING BIOMARKERS IN LYMPHANGIOLEIOMYOMATOSIS}

doi:10.1136/thx.2010.150938.8

W Y C Chang, L M Magowan, S R Johnson. University of Nottingham, Nottingham, UK

Rationale Lymphangioleiomyomatosis (LAM) is a rare disease of the lungs and lymphatics occurring almost exclusively in women, usually presenting before menopause. It is characterised by progressive cystic destruction of the lung parenchyma, obstruction of lymphatics, airways, and often progressive respiratory failure. Recent diagnostic criteria require lung biopsy in addition to HRCT in the absence of other features such as tuberous sclerosis, renal angiomyolipomas or chylous effusions. The clinical course of LAM varies significantly, and there are no good predictors of clinical progression. Vascular endothelial growth factor D (VEGFD) has been found to be increased in the serum of LAM patients but its role as a biomarker has never been examined prospectively.

We aimed to see if:

1. VEGF-D reduces the need for lung biopsy for diagnosis using a proposed cut off with an estimated test sensitivity for LAM of $86 \%$, specificity of $91 \%$, and a positive likelihood ratio of 9.6.

2. VEGF-D is a useful predictor of severity when correlated with lung function data.

Results Serum samples were taken from 34 LAM patients and 12 healthy controls and a significant difference in VEGF-D levels was seen (Patient median $=768 \mathrm{pg} / \mathrm{ml}, \quad \mathrm{IOR}=417.6-1509$, control median $=329.5, I O R=288.6-489.0, p=0.0082$, Mann-Whitney $U$ test). However, using the proposed cut off of $574 \mathrm{pg} / \mathrm{ml}$ by Young et al (2008), only 1 patient in our cohort with a diagnosis of "probable" LAM would have avoided the need for a lung biopsy to confirm diagnosis. When correlated with lung function, only TLCO demonstrated a statistically significant negative correlation with VEGFD levels $\left(r^{2}=0.2143, p=0.0131\right)$.

Conclusions Though an interesting research tool, the value of VEGFD as a biomarker in LAM has not been clearly demonstrated and there is currently insufficient evidence to advocate its role either to aid in diagnosis or prediction of outcome in LAM.

\section{S58 PULMONARY LANGERHANS' CELL HISTIOCYTOSIS (PLCH): A NEW NATIONAL REGISTER}

doi:10.1136/thx.2010.150938.9

${ }^{1} \mathrm{R}$ H Mason, ${ }^{2} \mathrm{~N}$ M Foley, ${ }^{1} \mathrm{M}$ R Hetzel, ${ }^{1} \mathrm{H}$ Branley, ${ }^{2} \mathrm{~J}$ Suntharalingam. ${ }^{1}$ Oxford Sleep Unit, The Churchill Hospital, Oxford, UK; ${ }^{2}$ Respiratory Department, Royal United Hospital, Bath, UK

Introduction and Objectives PLCH is a rare interstitial lung disease, linked to cigarette smoking and, in some cases associated with respiratory failure and death. Limited UK data have been published and hence little knowledge exists of the diagnostic and treatment practices employed by UK Respiratory physicians. Our study aims to characterise the epidemiological, clinical, histological, radiological and prognostic indicators in a nation wide cohort of patients with PLCH. This study aims to follow the patient journey from point of diagnosis, regardless of the mode of diagnosis, looking at patient demographics and clinical outcomes in the UK population.

Methods UK Respiratory Consultants were contacted to request details for 71 patients with known PLCH, who had previously been registered on the BTS Orphan Lung Disease (BOLD) database. Patients were sent a consent form and questionnaire. Once written consent was obtained, consultants were sent a questionnaire requesting medical information. The patients' GPs have provided current medication and past medical history information.

Results Details on 55 patients were received (including 10 deceased and, 4 lost to follow-up). 17 patients ( 8 males), have returned a completed consent form and questionnaire so far, and were included for analysis. Age at presentation 32.0 years (SD13.7). Presenting symptoms: Shortness of breath $70.5 \%$, pain $47.1 \%$, pneumothorax $11.8 \%$, cough $29.4 \%$ and $5.9 \%$ asymptomatic (diagnosed incidentally). Smoking status: Ex $88.2 \%$, current $6.0 \%$ mean (SD) 22 (27.2) pack years, and 6.0\% never smokers. $23.5 \%$ admitted limited exposure to cannabis. Diagnosis: $82 \%$ patients had had an HRCT scan and $64.7 \%$ an open lung biopsy. Diseasecourse: Symptoms resolved $35.3 \%$, same $41.2 \%$ and $23.5 \%$ had slowly progressed. $47.0 \%$ pneumothorax, $53.0 \%$ have received treatment either chemotherapy 29.4\% (Chlorambucil (2), 2-Chlorodeoxyadenosine (2), Azathioprine (1)) or, an operation for recurrent pneumothorax (23.5\%) 1 patient is on the waiting list for a lung transplant. Patients' opinion of Doctors knowledge of PLCH: High 35.3\%, medium 23.5\%, Med/low or low $41.1 \%$.

Conclusions Although small, this early dataset indicates a high prevalence of smoking in our UK cohort and, that despite advances in CT a high percentage of patients are still diagnosed with an open lung biopsy.

\section{a1-Antitrypsin: what it tells us about COPD S59 A A-VAL360: A PLASMA MARKER OF NEUTROPHIL ELASTASE ACTIVITY AND COPD DISEASE SEVERITY}

doi:10.1136/thx.2010.150938.10

${ }^{1} \mathrm{R}$ Carter, ${ }^{2} \mathrm{M}$ J Ungurs, ${ }^{1} \mathrm{R}$ A Stockley. ${ }^{1}$ Queen Elizabeth Hospital Birmingham, Birmingham, UK; ${ }^{2}$ University of Birmingham, Birmingham, UK

In both A1AT deficient and replete subjects, it is widely believed that protease-antiprotease imbalance is central to the pathophysiology of COPD, ${ }^{1}$ although (in the absence of an exacerbation) it is difficult to detect free elastase activity. ${ }^{2}$ This is explained by recent mathematical and in vitro modelling suggesting proteolysis and subsequent enzyme inhibition occur within the immediate microenvironment of the neutrophil. ${ }^{3}$ We have therefore validated a (preinhibition) elastase cleavage product of fibrinogen $\left(\mathrm{A} \alpha-\mathrm{Va}^{360}\right)$ produced in this microenvironment as a potential marker for COPD Methods Pilot study: Plasma A $\alpha-\mathrm{Val}^{360}$ was measured in 68 subjects with a wide range of A1AT levels, and in a further 64 PiZ A1AT deficient subjects, spirometry and plasma $\mathrm{A} \alpha-\mathrm{Val}^{360}$ were measured in the stable state. Subjects with COPD (Normal A1AT levels): 81 subjects were recruited in a primary care setting following an exacerbation associated with sputum production. Trial participants were assessed on day 1 of the exacerbation and in the stable clinical state when full lung function tests and HRCT were also performed. HRCT scans were analysed both densitometrically (voxel index) and visually. 